\title{
Associative Plasticity in the Medial Auditory Thalamus and Cerebellar Interpositus Nucleus during Eyeblink Conditioning
}

\author{
Hunter E. Halverson, Inah Lee, and John H. Freeman \\ Department of Psychology, University of Iowa, Iowa City, Iowa 52242
}

\begin{abstract}
Eyeblink conditioning, a type of associative motor learning, requires the cerebellum. The medial auditory thalamus is a necessary source of stimulus input to the cerebellum during auditory eyeblink conditioning. Nothing is currently known about interactions between the thalamus and cerebellum during associative learning. In the current study, neuronal activity was recorded in the cerebellar interpositus nucleus and medial auditory thalamus simultaneously from multiple tetrodes during auditory eyeblink conditioning to examine the relative timing of learning-related plasticity within these interconnected areas. Learning-related changes in neuronal activity correlated with the eyeblink conditioned response were evident in the cerebellum before the medial auditory thalamus over the course of training and within conditioning trials, suggesting that thalamic plasticity may be driven by cerebellar feedback. Short-latency plasticity developed in the thalamus during the first conditioning session and may reflect attention to the conditioned stimulus. Extinction training resulted in a decrease in learning-related activity in both structures and an increase in inhibition within the cerebellum. A feedback projection from the cerebellar nuclei to the medial auditory thalamus was identified, which may play a role in learning by facilitating stimulus input to the cerebellum via the thalamo-pontine projection.
\end{abstract}

\section{Introduction}

Pavlovian eyeblink conditioning is a type of associative learning that requires the anterior interpositus nucleus (IPN) and cortex of the cerebellum (Christian and Thompson, 2003; Ohyama et al., 2006). Unit activity in the IPN shows a learning-related increase during the conditioned stimulus (CS) that precedes the onset of the conditioned response (CR) (McCormick et al., 1982; McCormick and Thompson, 1984a,b; Berthier and Moore, 1990; Nicholson and Freeman, 2002; Choi and Moore, 2003; Green and Arenos, 2007). The increase in IPN activity develops only during forward paired presentations of the CS and unconditioned stimulus (US) and shows a rapid decline during extinction (Gould and Steinmetz, 1996).

Learning-related plasticity in the cerebellum develops because of convergent CS and US inputs in cerebellar cortex and IPN from the pontine nuclei (PN) and dorsal accessory inferior olive, respectively (Mauk et al., 1986; Steinmetz et al., 1987, 1989). Learning-related activity also develops in the PN during eyeblink conditioning, which is driven by excitatory feedback from the IPN and red nucleus (Cartford et al., 1997; Clark et al., 1997; Bao et al., 2000). Learning-related cerebellar feedback is also evident in the hippocampus (Clark et al., 1984; Sears and Steinmetz, 1990). However, the significance of IPN excitatory feedback to

\footnotetext{
Received Jan. 13, 2010; revised April 20, 2010; accepted May 17, 2010. This work was supported by National Institutes of Health Grant MH-080005 (J.H.F.).

Correspondence should be addressed to John H. Freeman, Department of Psychology, University of lowa, lowa City, IA 52242. E-mail: john-freeman@uiowa.edu.

DOI:10.1523/JNEUROSCI.0208-10.2010

Copyright $\odot 2010$ the authors $\quad 0270-6474 / 10 / 308787-10 \$ 15.00 / 0$
}

the PN and hippocampus for cerebellar learning is an unresolved issue.

The medial auditory thalamic nuclei (MATN), consisting of the medial division of the medial geniculate $(\mathrm{MGm})$, posterior intralaminar nucleus (PIN), and suprageniculate (SG), are a necessary part of the CS pathway for delay eyeblink conditioning with an auditory CS (Halverson and Freeman, 2006). These nuclei receive input from the shell of the inferior colliculus, cochlear nuclei, nucleus of the lateral lemniscus, superior olive, and superior colliculus (Calford and Aitkin, 1983; LeDoux et al., 1985, 1987; Malmierca et al., 2002). Single-unit recordings in the MGm during retention of differential trace eyeblink conditioning demonstrated increases in activity during reinforced trials and on trials when a CR is produced (O'Connor et al., 1997). The time course of learning-related changes in MATN activity during acquisition or extinction of eyeblink conditioning has not been investigated.

Neurons in the MATN respond to both auditory CSs and aversive USs and show associative plasticity during various conditioning procedures (Disterhoft and Olds, 1972; Gabriel et al., 1975; Ryugo and Weinberger, 1978; Supple and Kapp, 1989; Edeline and Weinberger, 1992; Bordi and LeDoux, 1994). Learning-related changes in activity within the MGm during pavlovian fear and discriminative avoidance conditioning depend on feedback from the amygdala (Maren et al., 2001; Poremba and Gabriel, 2001).

The current study examined simultaneous recordings from multiple tetrodes in the IPN and MATN during delay eyeblink conditioning and extinction. Based on previous demonstrations of cerebellar feedback to the PN and hippocampus, it is possible that learning-related increases in activity within the cerebellum 
drive learning-related activity in the MATN. This possibility was also examined using retrograde labeling of cerebellar projections to the MATN.

\section{Materials and Methods}

Subjects. The subjects were 16 male Long-Evans rats (250-400 g). The rats were housed in the animal colony in Spence Laboratories of Psychology at the University of Iowa (Iowa City, IA). All rats were maintained on a $12 \mathrm{~h} \mathrm{light/dark} \mathrm{cycle} \mathrm{and} \mathrm{given} \mathrm{ad} \mathrm{libitum} \mathrm{access} \mathrm{to} \mathrm{food} \mathrm{and} \mathrm{water.}$

Surgery. One week before the start of recording, rats were removed from their home cages and anesthetized with isoflurane. After the onset of anesthesia, electromyograph (EMG) electrodes (stainless steel) were implanted into the upper left orbicularis oculi muscle. The EMG electrode leads terminated in gold pins in a plastic connector. A bipolar stimulating electrode for delivering the shock US was implanted subdermally, caudal to the left eye. During the same surgery, a custom-made hyperdrive array fitted with an electronic interface board (Neuralynx) was implanted over the left IPN and right MATN. The IPN ipsilateral and MATN contralateral to the eye receiving the US are necessary for delay eyeblink conditioning in rats (Freeman et al., 2005; Halverson and Freeman, 2006). Learning-related changes in neuronal activity that could play a role in production of the eyeblink conditioned response were, therefore, expected to be seen in the left IPN and right MATN. Stereotaxic coordinates taken from bregma for the IPN were $11.4 \mathrm{~mm}$ posterior, $2.9 \mathrm{~mm}$ lateral, and $5.6 \mathrm{~mm}$ ventral to the skull surface. Stereotaxic coordinates taken from bregma for the MATN (MGm) were $5.5 \mathrm{~mm}$ posterior, $3.1 \mathrm{~mm}$ lateral, and $6.3 \mathrm{~mm}$ ventral to the skull surface. The skull surface was marked for each tetrode bundle and drilled out with a square blunt drill bit. Skull fragments were carefully removed along with the dura matter for each drill hole under visual guidance. Bundles of tetrodes were lowered to the surface of the brain and sealed with low viscosity silicon (Kwik-Sil; World Precision Instruments). Three tetrodes in one bundle (three recording tetrodes, one reference wire) were lowered to the MATN and another bundle (three recording tetrodes, one reference wire) was lowered to the IPN. Reference tetrodes were lowered so each reference was $0.5 \mathrm{~mm}$ above the IPN and MATN, respectively. Each independently moveable tetrode was composed of four nichrome wires (12 $\mu \mathrm{m}$ diameter; Kanthal Palm Coast), twisted and partially melted together to form a tetrode. Each tetrode was gold plated to reduce final impedance to $250-500 \mathrm{k} \Omega$ measured at $1 \mathrm{kHz}$ (impedance tester IMP-1; Bak Electronics).

Recording and unit isolation. Tetrode bundles were placed over the IPN and MATN, and tetrodes were advanced to within $2.0 \mathrm{~mm}$ of the target nuclei during surgery using stereotaxic guidance. Tetrodes were lowered to the target nuclei while changes in neuronal activity were observed in both IPN and MATN. IPN neuronal activity emerged as the tetrode advanced through the white matter and into the nucleus. Neuronal activity in the MATN was found in much the same way, although auditory stimuli (clap, tapping on chamber, whistle) would often evoke a response from activity being monitored in the auditory thalamus before conditioning. The head stage (Neuralynx) was connected to a motorized commutator to allow the rats to move during training sessions. After stable units were obtained, eyeblink training commenced along with neural recording. Neuronal signals were first preamplified at unity-gain. The signals were then amplified between 2000 and 20,000 times and bandpass filtered $(0.3-6 \mathrm{kHz}$; Neuralynx). Neural signals that exceeded a channel amplitude threshold were digitized and stored at $32 \mathrm{kHz}$ (Cheetah system; Neuralynx). Waveform characteristics were plotted as a scatterplot of one of the electrodes versus another in the energy, peak, and valley planes. The energy presentation represents the square root of the sum of the squared points for the entire waveform. The peak presentation is the maximum height (positive amplitude) of the waveform in each channel of the spike. The valley presentation is the maximum depth (negative amplitude) of the waveform of each channel of the spike (MClust). Individual units formed clusters of points on these different presentations, and boundaries of these plots were defined under visual inspection with an interactive spike sorting software (MClust). An automatic cluster cutting program was used (Klusta-Kwik) to identify possible clusters followed by additional manual identification of single units.

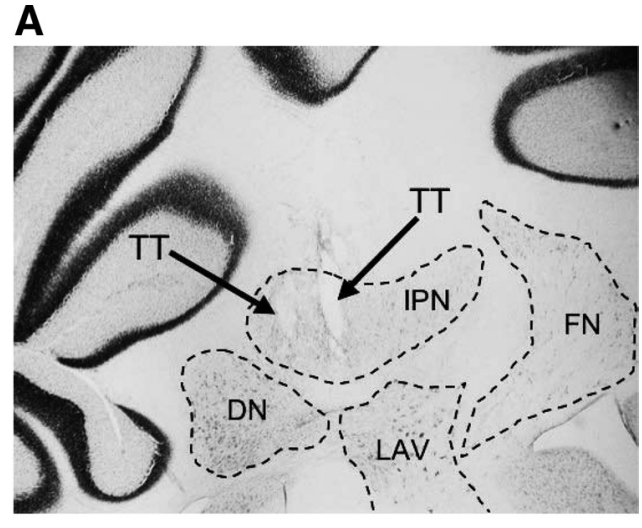

B

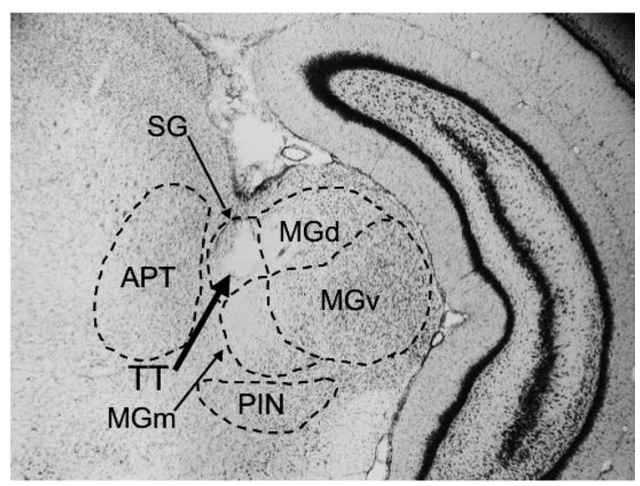

Figure 1. A, Coronal section of the cerebellum showing tetrode placements in the anterior IPN. An additional tetrode immediately posterior to the right tetrode contributed to the size of the marking lesion. DN, Dentate nucleus; FN, fastigial nucleus; LAV, lateral vestibular nucleus; TT, tetrode tip. Magnification, $2.5 \times$. B, Coronal section of the thalamus showing a representative tetrode placement in the MATN (SG). APT, Anterior pretectal nucleus; MGd, dorsal division of the medial geniculate; $M G v$, ventral division of the medial geniculate; $M G m$, medial division of the medial geniculate; PIN, posterior intralaminar nucleus; $\mathrm{SG}$, suprageniculate; TT, tetrode tip. Magnification, $2.5 \times$.

Single-unit data analysis. Peristimulus histograms of unit activity for the IPN and MATN were created for both CS and US periods (366 ms). Stimulus periods were divided into three equal blocks and each block was divided into 10 equal bins (122 ms blocks; $12.2 \mathrm{~ms}$ bins). Analysis of the unit activity for each block during the CS and US for all trials was compared with an equal pre-CS baseline period (122 ms) using the Wilcoxon signed-rank matched-pairs test (Kubota et al., 1996; Freeman and Nicholson, 1999, 2000; Nicholson and Freeman, 2002, 2003a,b). Wilcoxon's tests were also done on the initial $50 \mathrm{~ms}$ of the CS period to better classify the different onset responses observed in the MATN. Different response types were classified based on the results of the Wilcoxon tests showing significant differences in unit responses compared with the baseline period. Additional Wilcoxon's tests were done on each unit for each session during the CS and US periods for CR and non-CR trials to identify differences in neural responding that were correlated with performance of the conditioned response. Percentage differences in activity between $\mathrm{CR}$ and non-CR trials during different stimulus blocks were analyzed with the $\chi^{2}$ test. The Wilcoxon test is a nonparametric method to determine significance for increases and decreases in activity without assumptions about the distribution of the data points but does not provide information about the magnitude of change. Normalization was used to assess the magnitude of changes in neuronal activity. Neuronal activity in each bin within the CS was normalized to the baseline activity for each unit (Freeman and Nicholson, 1999, 2000; Nicholson and Freeman, 2002). To assess the neural-behavioral timing specificity between unit activity in the IPN and MATN and eyelid EMG during eyeblink conditioning, cross-correlations were calculated between each rat's EMG activity and each unit's cumulative activity time locked to the onset of the 
A

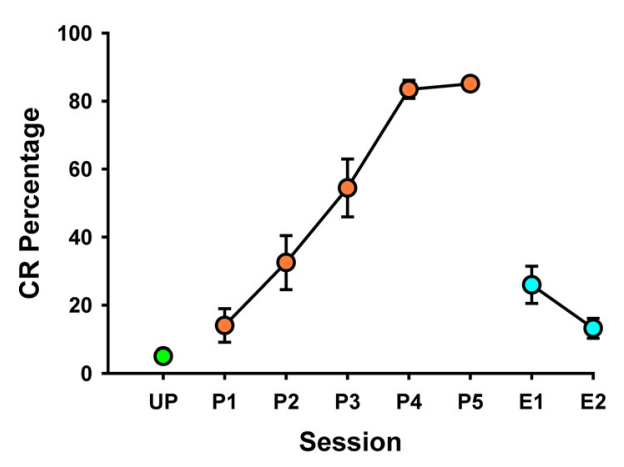

B

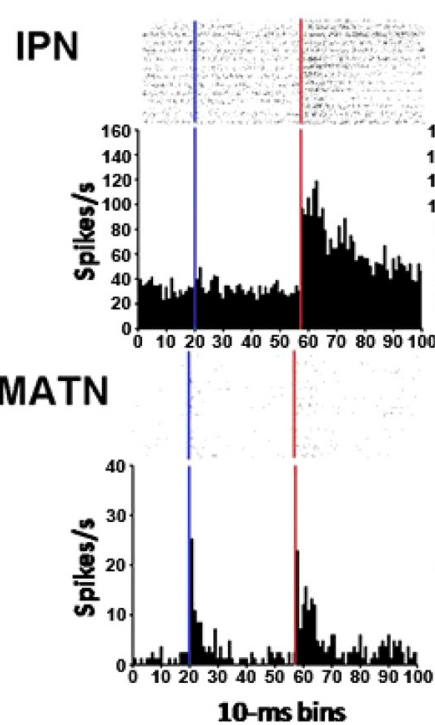

P1

P3

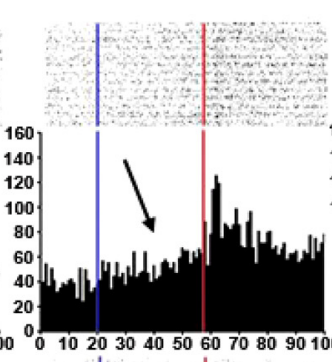

P5

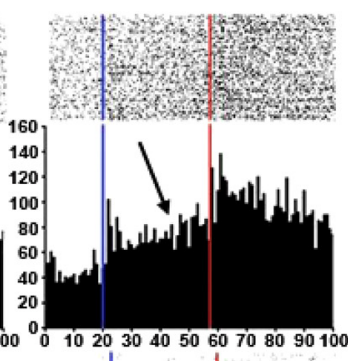

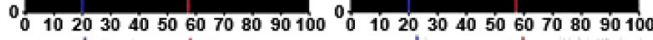

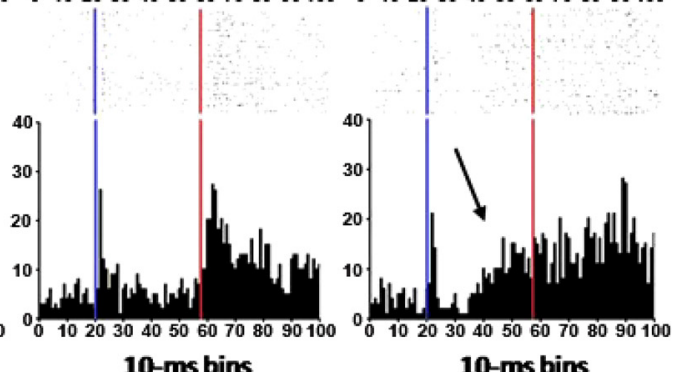

10-ms bins

10-ms bins

Figure 2. A, Mean $\pm \mathrm{SE}$; CR percentage for rats during each session of training. UP, Unpaired (green); P1-P5, paired 1-5 (orange); E1-E2, extinction (teal). B, Histograms with raster plots of single unit activity (spikes/second) in anterior IPN and MATN during the first paired (P1), third paired (P3), and fifth paired (P5) sessions. Learning-related unit activity (arrows) increased in the IPN from P1 to P5. Learning-related unit activity is evident in the MATN on P5. The blue line represents CS onset, and the red line represents US onset. The bars in the histograms are $10 \mathrm{~ms}$.

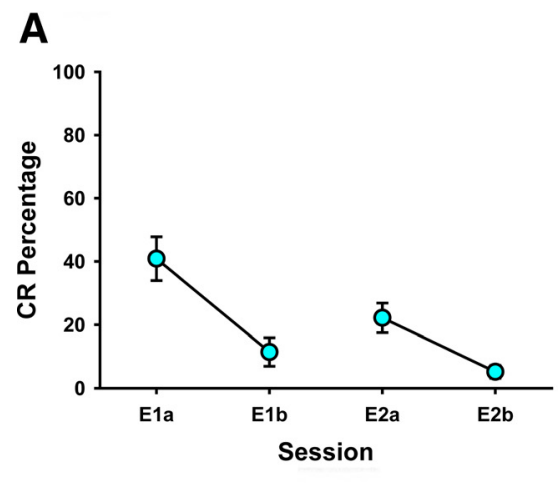

B
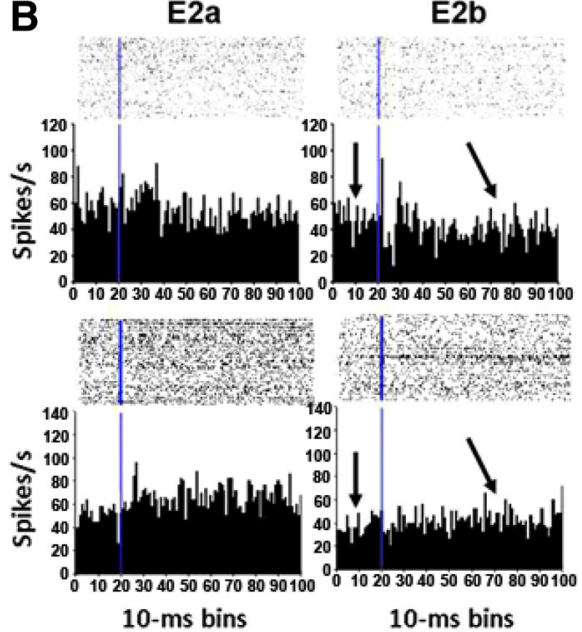

Figure 3. $A$, Mean $\pm \mathrm{SE}$; CR percentage for rats during extinction sessions. E1a, E2a, First 50 trials; E1b, E2b, last 50 trials. $B$, Histograms with raster plots of unit activity (spikes/second) for two anterior interpositus nucleus units during E2a,b. Both units showed a progressive decrease in pre-CS baseline (arrows) during extinction. Both units also showed an increase in inhibitory responses during the CS period on E2 (arrows). Each row follows a single IPN unit through E2. The blue line represents CS onset. The bars in the histograms are $10 \mathrm{~ms}$.

CR for CS-alone trials during the final paired session (session 5). Crosscorrelations were also calculated between average EMG activity and the mean population unit responses in IPN and each area of the MATN. Spike-to-spike cross-correlations were calculated between simultaneously recorded units in the MATN and IPN.

Conditioning procedure. The tone CS used in training was a $391 \mathrm{~ms}$, $2000 \mathrm{~Hz}$ pure tone $(85 \mathrm{~dB}$; range in conditioning chamber, $83-87 \mathrm{~dB})$. All animals initially received one session of explicitly unpaired presentations of the tone CS and a $1.0 \mathrm{~ms}$ shock US to obtain a baseline of unit responses to stimuli used during conditioning. Rats then received five sessions of paired delay eyeblink conditioning with the CS and a $25 \mathrm{~ms}$ shock US to obtain unit response during acquisition and expression of CRs. Paired sessions were followed by two extinction sessions in which rats were presented CS-alone trials. Each of the sessions of delay eyeblink conditioning consisted of 100 trials per day with a pseudorandom distribu- tion of intertrial intervals between 18 and $42 \mathrm{~s}$ that averaged $30 \mathrm{~s}$. The $391 \mathrm{~ms}$ CS coterminated with a $25 \mathrm{~ms}$ shock US, yielding an interstimulus interval of $366 \mathrm{~ms}$. Daily training sessions consisted of 10 blocks of 9 paired CS-US presentations and a CS-alone trial. Extinction sessions were similar to paired sessions except all 100 trials were CS-alone presentations.

Histology. After completion of the experiment, small marker lesions were made on the tetrode tips $1 \mathrm{~d}$ before perfusion. Brains were sliced and mounted on slides and sections were then stained with thionin. The final placement of each tetrode was verified using a light microscope (Leica DMLS) and a stereotaxic brain atlas (Paxinos and Watson, 1998).

Retrograde labeling with Fluoro-Gold. Rats $(n=5)$ were removed from their home cage and anesthetized with isoflurane. A 32 gauge infusion cannula was then lowered into the MATN. The stereotaxic coordinates taken from bregma for the cannula were $5.5 \mathrm{~mm}$ posterior, $3.1 \mathrm{~mm}$ lateral, and $6.1 \mathrm{~mm}$ ventral to the skull surface. After $5 \mathrm{~min}, 0.05 \mu \mathrm{l}$ of $4.0 \%$ Fluoro-Gold (Fluorochrome) was infused into the MATN at a rate of $1.0 \mu \mathrm{l} / \mathrm{h}$ over $3 \mathrm{~min}$. The postinfusion survival time was $14 \mathrm{~d}$. Brains were sectioned at $50 \mu \mathrm{m}$, and slices from auditory structures, brainstem, and cerebellum were mounted on slides. The Fluoro-Gold infusion site in MATN and subsequent retrograde labeling was then investigated with a Nikon Diaphot 300 microscope and filter set.

\section{Results}

\section{Tetrode placement}

Results from 1151 units recorded from 12 rats were analyzed; 876 were located in the MATN (SG, 235; MGm, 441; PIN, 200), and 275 in the IPN. A recording from a tetrode in the IPN or MATN was included in the analysis as a single unit if the unit was distinguishable from other putative units recorded by the same tetrode and if the tetrode placement was verified in the IPN or MATN 
after the experiment. Tetrodes in the cerebellum were located in the anterior interpositus nucleus $(n=23)$ (Fig. $1 A)$. All IPN units were spontaneously active with an overall average firing rate of $56.7 \mathrm{~Hz}$. Missed tetrodes were dorsal $(n=5)$ or ventral to IPN $(n=1)$. Data from missed tetrodes in the cerebellum were not included in the final analysis. Reference electrodes in the cerebellum were located dorsal to the recording tetrodes in the white matter immediately dorsal to the IPN $(n=6)$. In some cases, reference electrodes in MATN were used as the IPN reference. Tetrodes in the MATN were located in MGm $(n=15), \operatorname{PIN}(n=6)$, and SG $(n=9)$ (Fig. $1 B)$. MATN units were also spontaneously active but at a much lower frequency than observed in IPN (MGm, 7.1 Hz; SG, 11.4 Hz; PIN, 9.9 $\mathrm{Hz}$ ). Units that were not spontaneously active but responded to auditory stimuli were also observed (Bordi and LeDoux, 1994). One tetrode targeting the MATN was ventral to the PIN, and data from this tetrode were not used in the analyses. Reference electrodes in the thalamus were located in the dorsal division of the medial geniculate $(n=5)$, ventral division of the medial geniculate $(n=2)$, anterior pretectal nucleus $(n=3)$, and lateral posterior nucleus of the thalamus (mediocaudal region) $(n=1)$.

\section{Eyeblink conditioning}

All of the rats showed a progressive increase in CRs during the five paired sessions relative to the unpaired session $(p<0.001)$ (Fig. $2 A)$. The acquisition observed during the paired sessions was similar to previous studies using a tone CS (Halverson and Freeman, 2006; Halverson et al., 2008). Rats also showed a substantial decrease in CRs during the two extinction sessions relative to the final session of paired training $(p<0.001)$ (Figs. $2 A, 3 A)$. The rapid loss of CRs during extinction training was also similar to a previous study (Nolan and Freeman, 2006).

\section{Stimulus-elicited activity}

Peristimulus histograms of unit activity time locked to the beginning of each trial for the IPN and MATN for the unpaired pretraining session (UP), first paired session (P1), third paired session (P3), fifth paired session (P5), and both extinction sessions (E1, E2) were examined to classify discharge patterns during the CS and US. Initial classifications were made for unit responses obtained during the UP session to examine activity during the CS and US before conditioning (supplemental Fig. S1, supplemental data, available at www.jneurosci.org as supplemental material). All comparisons of the percentage of unit response types were made with the $\chi^{2}$ test at the $p<0.05$ significance level. Units in IPN and MATN showed a change (increase or decrease) in activity to the CS and US relative to the baseline during unpaired training (IPN, 42\%/82\%; MATN, 66\%/ $71 \%)$. CS-related unit responses in IPN and MATN showed an increase over the course of acquisition training (Fig. 2 B). A shortlatency increase in CS-elicited activity that emerged during the first training sessions was observed in the MGm, a finding that is consistent with the results of a previous fear conditioning study (Maren et al., 2001) (supplemental Fig. S2, supplemental data, available at www.jneurosci.org as supplemental material). Extinction training resulted in the loss of learning-related responses in IPN and MATN, and a decrease in IPN baseline activity during the second half of each extinction session (Fig. $3 B$ ). The detailed findings from the stimulus elicited activity are presented in the supplemental data (available at www.jneurosci.org as supplemental material).

\section{CR-related activity}

The percentage of units that showed significant increases or decreases from pre-CS baseline activity during CR and non-CR trials was examined for each area during P1, P3, P5, E1, and E2 to assess changes in CR-related activity. The percentage of units showing differences from pre-CS activity was also compared with data from the unpaired session (UP) to investigate learningrelated changes in unit responses relative to a nonassociative baseline. Histograms of unit activity in the IPN and MATN in the CS period during UP and also for CR and non-CR trials during the training sessions were divided into three $122 \mathrm{~ms}$ periods ( 10 blocks of $12.2 \mathrm{~ms}$ ) and compared with an equal time period from the pre-CS baseline with the Wilcoxon test (Kubota et al., 1996; Freeman and Nicholson, 1999, 2000; Nicholson and Freeman, 2002, 2003a,b). Three CS periods were used for the analysis to determine when learning-related changes occurred during the $\mathrm{CS}$, and also to examine the development of neural plasticity as learning progressed. All comparisons of the percentage of units 

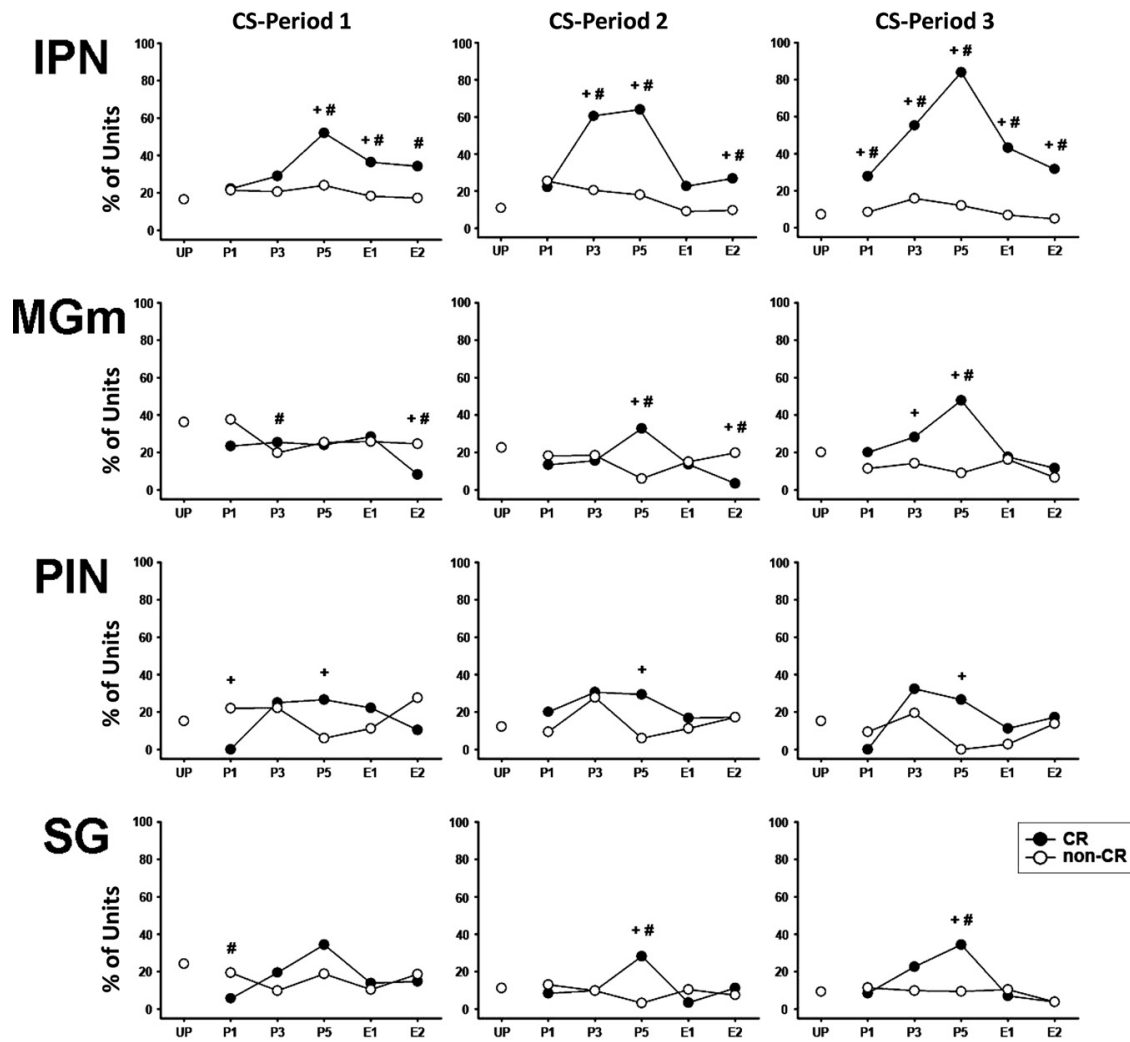

\section{Training Sessions}

Figure 5. Percentage of single units in the anterior IPN and MATN showing significant increases, relative to pre-CS baseline, during CR trials (black) and non-CR trials (white) for the three CS periods during the unpaired session (UP), first (P1), third (P3), and fifth (P5) paired sessions, and two extinction sessions (E1, E2). ${ }^{+}$Significant differences between CR and non-CR trials; ${ }^{\#}$ percentage is different from UP level.

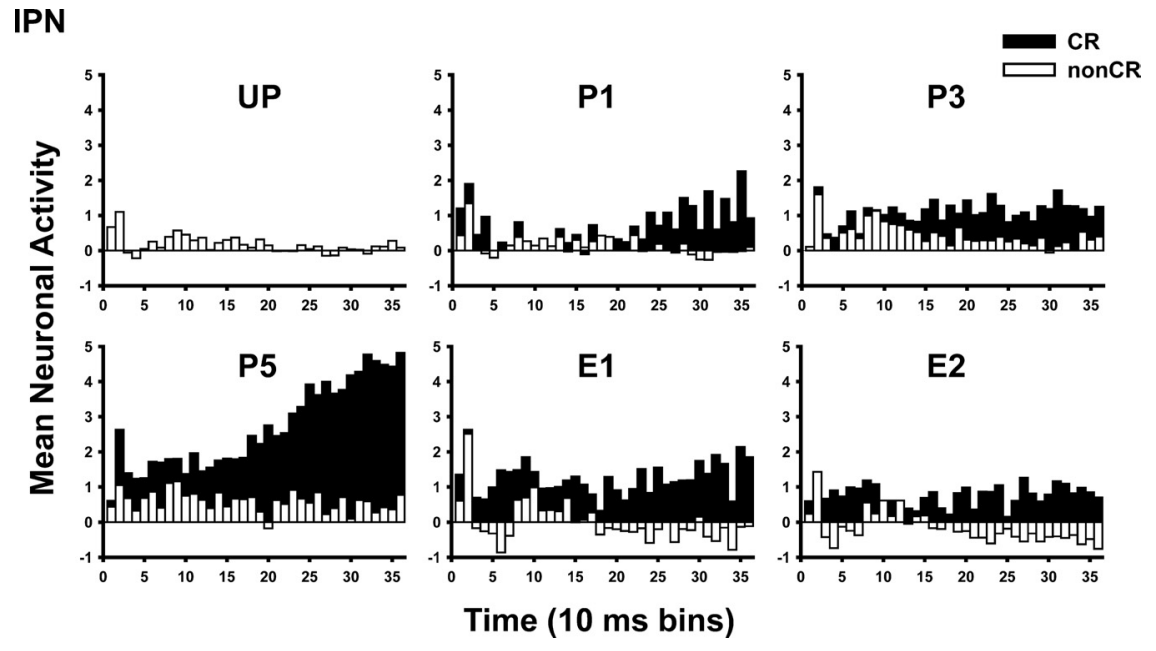

Figure 6. Mean normalized unit activity recorded from the anterior IPN during the unpaired session (UP), first paired (P1), third paired (P3), fifth paired (P5), first extinction (E1), and second extinction (E2) sessions for trials with and without an eyeblink $C R$.

between CR and non-CR trials and from the UP baseline were made with the $\chi^{2}$ test.

\section{CR-related activity in the IPN}

An increase in learning-related activity in IPN that paralleled the increase in CR percentage was observed during acquisition training, and decreased, along with the decrease in CR percentage,

during extinction training. Figure 4 shows single-unit responses in the IPN and MATN during CR and non-CR trials on P5. Units in the IPN showed an increase in activity on trials with CRs relative to trials without CRs that preceded the onset of the CR within trials (McCormick and Thompson, 1984a,b; Berthier and Moore, 1990; Freeman and Nicholson, 2000; Nicholson and Freeman, 2002; Choi and Moore, 2003; Green and Arenos, 2007). MATN activity also increased during trials with CRs, but this increase in activity typically followed the increase in IPN activity within trials (Fig. 4). Figure 5 shows the percentage of single units with significant increases in activity across the three time periods during the CS in the IPN and MATN for CR and non-CR trials during P1, P3, P5, and both extinction sessions relative to activity during the unpaired session. The percentage of IPN units showing a significant increase in activity during the CS was greater on CR trials $(27.8 \%)$ relative to non-CR trials $(8.5 \%)$ during $\mathrm{P} 1$, indicating that learning-related activity emerged very early in IPN. During acquisition training, the percentage of units showing a CR-related increase in activity progressively increased in parallel with CR percentage during P3 and P5 (Fig. 5). There was a decrease in the percentage of excitatory responses to the CS from E1 to E2 (52 to 29\%) and a decrease in baseline activity during the second half (last 50 trials) of $\mathrm{E} 1$ and $\mathrm{E} 2$ relative to the first half of each session (Fig. 3B). During E1, $50 \%$ of the IPN units showed a decrease in baseline activity and $53.6 \%$ of the units showed a decrease in baseline during E2. Extinction training also produced an increase in the percentage of units showing inhibitory responses during non-CR trials in the IPN (E1, 23\%; E2, 33\%) (Fig. 3B).

The magnitude of learning-related changes in unit activity in the IPN was examined for sessions UP, P1, P3, P5, E1, and E2 by normalizing CS-elicited responses to the pre-CS baseline for CR and non-CR trials. Differences between intervals and CR/non-CR unit responses across sessions were examined with a repeated-measures ANOVA, which revealed an interaction of the interval (10 $\mathrm{ms}$ ) and session (100 trials each) factors $\left(F_{(35,350)}=4.383 ; p<0.0001\right)$. The magnitude of unit activity was greater on CR trials relative to non-CR trials on P1 (intervals 4, 24, 26, 28, 29, 31, 33, 35), P3 (intervals 15, 16, 18, 20-24, 27-36), and P5 (intervals 2, 7, 11, 13-36), E1 (intervals 4-7, 9, 15, 16, 19, 20, 22-36), and E2 (intervals 3, 4, 6, 7, 16, 19, 20, 22-24, 26-36) (Fig. 6). These findings indicate that the magnitude of differences in activity between trials with CRs and no CRs increased 
as learning progressed and then decreased during extinction, although significant differences were still clearly evident during extinction.

\section{CR-related activity in the MGm}

Learning-related unit activity was evident later in training in the MGm relative to the IPN in terms of the percentage of units showing increased activity during CR trials. However, differences between $\mathrm{CR}$ and non-CR trials became more pronounced as training progressed. The percentage of units showing a significant increase in activity during the CS on CR trials (28.2\%) was greater than the percentage of units showing increased activity on non-CR trials (14.1\%) during P3 and this difference became more pronounced on P5 (47.8\% on CR trials vs $8.9 \%$ on non-CR trials). The learning-related activity in MGm also emerged slightly later than the increase in CR percentage. This pattern suggests that learning-related activity was established in the MGm after learning started. Unit responses returned to, or fell below, UP baseline levels during extinction training, along with the observed decrease in $\mathrm{CR}$ percentage.

Analysis of normalized activity from the MGm revealed earlier-developing plasticity than the analysis of the percentage of units with significant changes in activity presented above. A repeated-measures ANOVA yielded an interaction of the interval and session factors $\left(F_{(35,350)}=\right.$ $1.908 ; p<0.0001)$. Post hoc tests indicated greater unit activity on CR trials relative to non-CR trials during P1 (intervals 1, 3, 5-8, 18, 19, 21, 29, 31-33), P3 (intervals 2, $3,27,32,34,36$ ), and P5 (intervals 1-4, $28,31-33,35,36$ ) (Fig. 7). Activity in the MGm rapidly returned to baseline levels during extinction training.

\section{CR-related activity in the PIN and SG}

Unit activity in the PIN and SG did not show differences between $\mathrm{CR}$ and non-CR trials until P5, which was attributable to a decrease in the percentage of excitatory CS-elicited activity during non-CR trials. Conditioned response-related activity in the PIN and SG developed later in training relative to the IPN (P1) and MGm (P3). The activity of units in the PIN and SG returned to UP baseline levels during extinction, and no differences were observed in $\mathrm{CR}$ versus non-CR unit responses.

Analysis of the normalized activity in the PIN and SG revealed a different pattern of results than in the MGm. A repeatedmeasures ANOVA on the unit activity in the PIN revealed an interaction of the interval and session factors $\left(F_{(35,350)}=1.288\right.$;

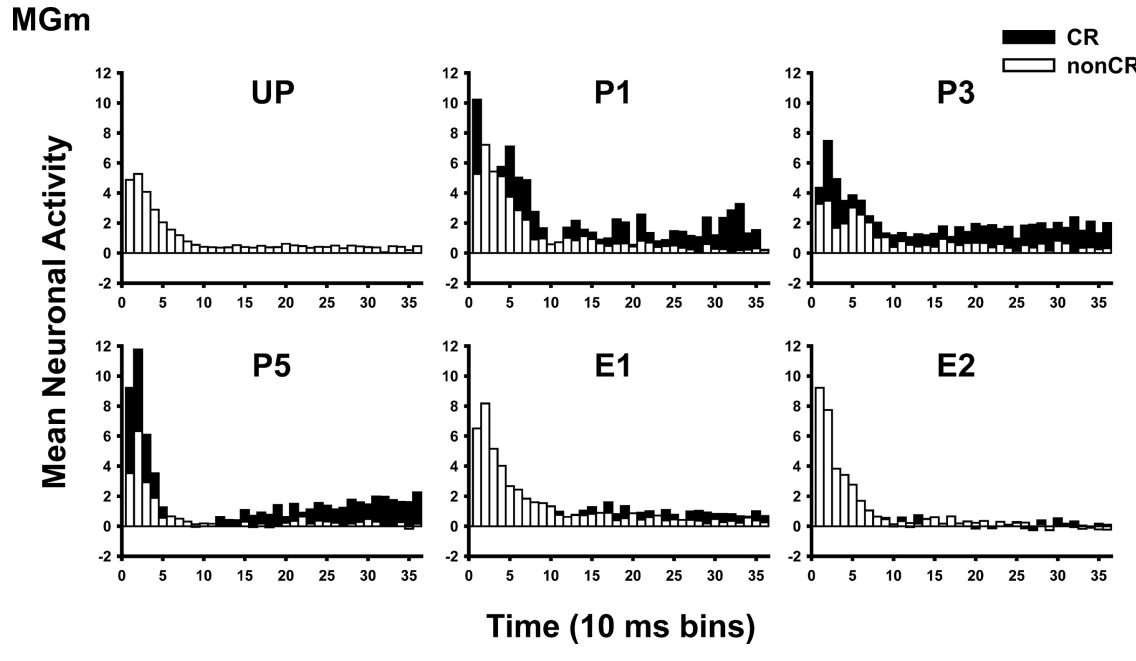

Figure 7. Mean normalized unit activity recorded from the MGm during the unpaired session (UP), first paired (P1), third paired (P3), fifth paired (P5), first extinction (E1), and second extinction (E2) sessions for trials with and without an eyeblink CR.
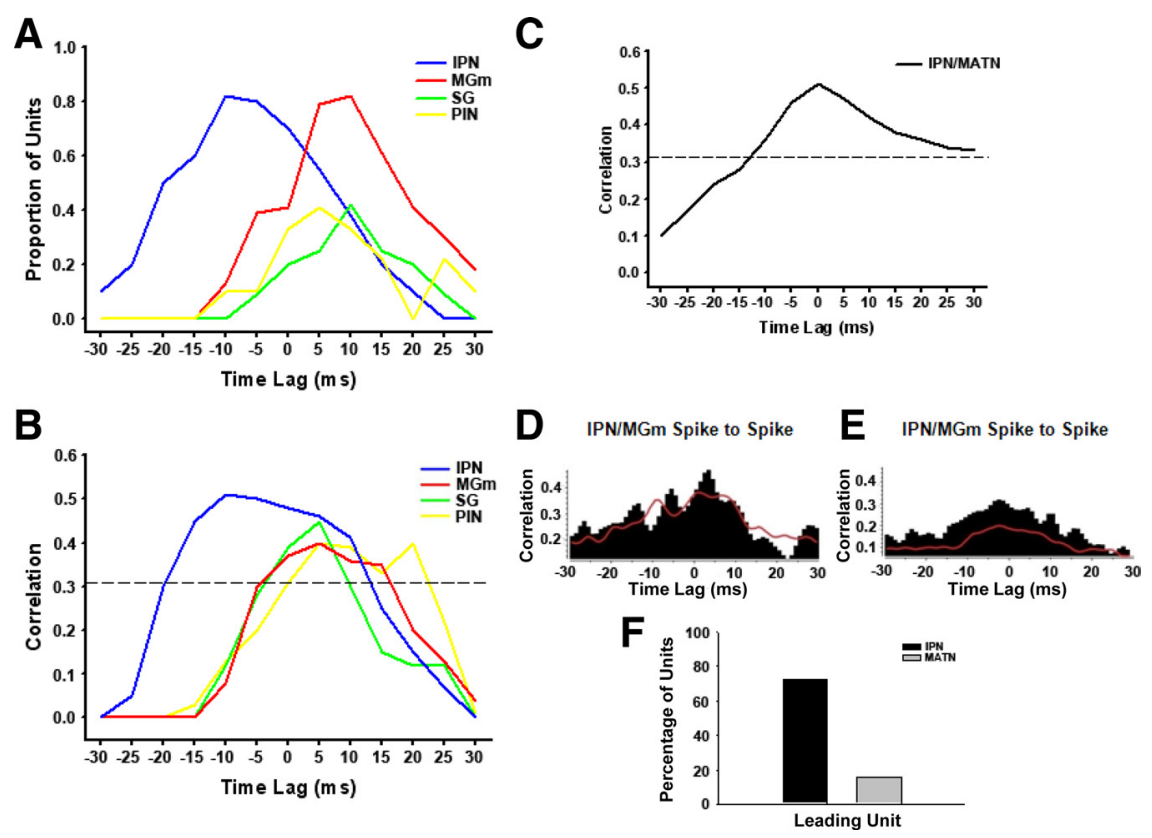

Figure 8. A, Line graph showing the proportion of units that showed significant cross-correlations with EMG activity time locked to CR onset (0 time lag) at different time lags in the anterior IPN (blue), MGm (red), SG (green), and PIN (yellow). There was a greater proportion of IPN units with positive correlations at earlier time lags relative to each area of MATN. $\boldsymbol{B}$, Line graph showing cross-correlations between average unit activity in the IPN and each area of MATN with average eyelid EMG activity time-locked to the onset of the CR for CS-alone trials in which a CR was performed during P5. Significant correlations (dotted line) for the population of IPN units showed a negative time lag of $-18 \mathrm{~ms}$, with the highest correlations between -12 and $-10 \mathrm{~ms}$, indicating IPN units showed an increase in activity before the onset of the CR. Significant positive correlations for the population of units for each area of MATN showed a negative time lag of $-5 \mathrm{~ms}$ indicating that MATN units fire just before the onset of the CR. The highest correlations were between time lags $5-10 \mathrm{~ms}$ after CR onset. C, Line graph showing cross-correlation between average learning-related unit activity in IPN and learning-related activity from each area of MATN time-locked to the onset of the CR. The highest significant correlations (dotted line) were between $-5 \mathrm{~ms}$ and $(R$ onset (time lag, 0). D, E, Examples of spike-to-spike cross-correlations for individual simultaneously recorded units in MGm and IPN on P5 ( 0 , CR onset; MGm unit as reference). The highest correlations were before and immediately after $C R$ onset as observed with the population analysis. The red line indicates the shift predictor for the MGm unit relative to the IPN unit in each case. $F$, Bar graph showing the percentage of units in IPN or MATN showing cross-correlations with the earliest lead times relative to $C R$ onset when considering all possible combinations of simultaneously recorded units within animals. 
A
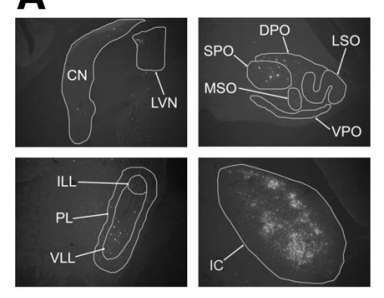

B

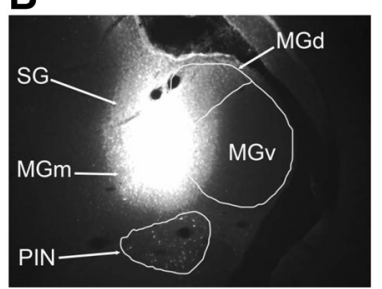

C

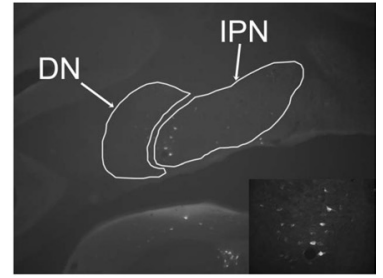

D

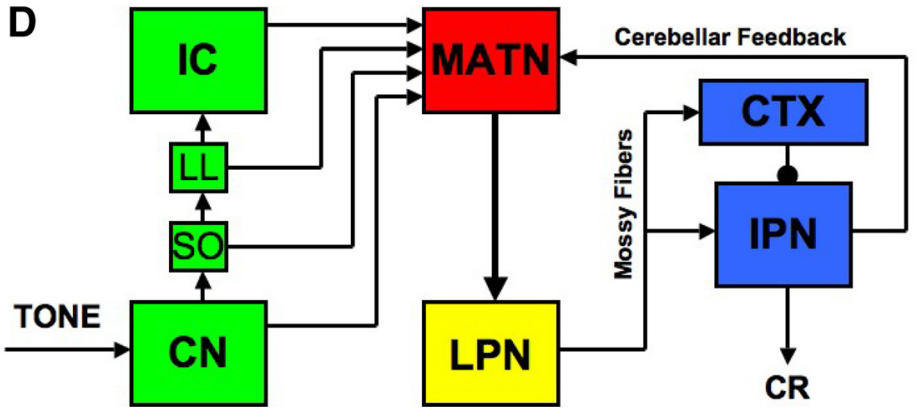

Figure 9. A, Coronal section of the lower brainstem and inferior colliculus (IC) showing labeled neurons in the cochlear nucleus (CN), lateral vestibular nucleus (LVN), dorsal periolivary region (DPO), medial superior olive (MSO), ventral periolivary nucleus (VPO), ventral nucleus of the lateral lemniscus (VLL), intermediate nucleus of the lateral lemniscus (ILL), and throughout the IC. LSO, Lateral superior olive; SPO, superior periolivary nucleus; DLL, dorsal nucleus of the lateral lemniscus. Magnification, $4 \times$. $\boldsymbol{B}$, Coronal section of the auditory thalamus showing the Fluoro-Gold infusion site. Labeled neurons are shown in the PIN. SG, Suprageniculate; MGm, medial division of the medial geniculate; MGd, dorsal division of the medial geniculate; MGv, ventral division of the medial geniculate. Magnification, $4 \times$.C, Coronal section of the cerebellum showing labeled neurons in the lateral portion of the anterior interpositus (IPN). Fluoro-Gold infusions in the anterior MATN produced labeled neurons in IPN, and infusions into posterior MATN produced labeled neurons in the dentate nucleus. Labeled neurons were contralateral to the infusion sites. Magnification, $4 \times$ and $20 \times$ for close-up of neurons. D, Hypothesized auditory CS pathway for eyeblink conditioning. Parallel inputs into the MATN from cochlear nucleus (CN), superior olive (SO), lateral lemniscus (LL), and inferior colliculus (IC). Auditory input is then projected to lateral pontine nuclei (LPN), which project to the cerebellum (cortex and interpositus nucleus). A feedback projection from the IPN to the MATN is also shown.

ANOVA on unit activity in the SG revealed a main effect of interval $\left(F_{(35,350)}=23.892 ; p<0.0001\right)$. Post hoc tests indicated differences in SG activity were confined to the first four intervals, with higher activity on CR trials during interval 1 on P1 and on intervals 1-4 during P5. Like the MGm and PIN, no differences were found in average unit activity during extinction in the SG.

\section{Temporal relationship of unit and eyelid EMG activity}

To determine the relationship between the timing of learningrelated unit responses in the IPN, MGm, PIN, and SG with the onset of the behavioral CR, cross-correlations were calculated between these variables during CS-alone trials in which the rat produced a CR. CS-alone trials were analyzed to examine correlations between unit activity and the conditioned blink response in the absence of the US and unconditioned response. Unit activity in the IPN has been shown to precede the onset of the behavioral CR by as much as $40-60 \mathrm{~ms}$ in rabbits while measuring the nictitating membrane (NM) response (McCormick and Thompson, 1984b). However, when the CR is measured using EMG, as in the current experiment, the onset of the response is $30 \mathrm{~ms}$ earlier than the NM response (McCormick et al., 1982). IPN unit responses in the current study should, therefore, show the highest correlations with the onset of the CR at negative time lags between 10 and $30 \mathrm{~ms}$. Unit responses in the MATN, particularly in the MGm, also showed an increase during the last CS period that was dependent on the presence of the CR. If the highest correlations between eyelid and MATN activity are at time lags during or after the onset of the CR that would indicate that the late responses in the MATN could be driven by feedback from the per- formance of the response or possibly by feedback from the cerebellum (IPN). Alternatively, if the late responses in the MATN show the highest correlations with the $\mathrm{CR}$ at negative time lags that occur before those observed in the IPN, that would indicate that the MATN could be driving activity in the IPN to produce the CR. Cross-correlations were also run between the population and single-unit data for IPN and each area of the MATN.

Cross-correlations calculated between unit activity in the IPN and EMG activity for each rat revealed that the largest proportion of units in the IPN showed significant positive correlations with EMG activity at a time lag of $-10 \mathrm{~ms}$ (Fig. 8A). A large proportion of units also showed positive correlations with EMG activity at time lags of -15 and $-20 \mathrm{~ms}$. Correlations at these time lags are consistent with previous studies examining IPN activity in rabbits and rats (McCormick and Thompson, 1984b; Green et al., 2002; Choi and Moore, 2003). A cross-correlation between average IPN activity and average EMG activity revealed significant correlations at negative time lags starting at -18 $\mathrm{ms}$, with the highest correlations between -10 to -12 ms before CR onset (Fig. $8 B$ ).

Cross-correlations between learningrelated unit activity in the MGm and EMG activity revealed significant correlations starting at $-10 \mathrm{~ms}$ relative to $\mathrm{CR}$ onset. The largest proportion of positive correlations was observed at time lags between +5 and $+10 \mathrm{~ms}$ (Fig. $8 \mathrm{~A}$ ). Positive correlations may be the result of feedback from the CR or cerebellum (IPN). Cross-correlations between average unit activity and average EMG activity revealed significant correlations at time lags starting at $-5 \mathrm{~ms}$, with the highest correlations between +10 and $+12 \mathrm{~ms}$ after CR onset (Fig. $8 \mathrm{~B}$ ).

Cross-correlations between CR-related unit activity in the PIN and SG, and EMG activity revealed the largest proportion of positive correlations between +5 and $+10 \mathrm{~ms}$, which was similar to the results observed in the MGm (Fig. $8 \mathrm{~A}$ ). Cross-correlations between average unit responses and average EMG activity also revealed significant correlations at time lags starting at $-5 \mathrm{~ms}$, with the highest positive correlations for the PIN at $+10 \mathrm{~ms}$, and $+5 \mathrm{~ms}$ for the SG (Fig. $8 B$ ).

The results of the cross-correlation analyses indicate that, at the individual unit and population levels, increases in unit activity in both the IPN and MATN preceded the onset of the CR with activity in the IPN occurring before the MATN. The significant positive correlations at time lags close to CR onset in the MATN suggest that this activity could be driven by direct or indirect feedback from the cerebellum. The earliest and highest correlations with the CR were in the IPN. The highest correlations in IPN were before CR onset, whereas the highest correlations in each area of MATN were after CR onset. Cross-correlations between average IPN and MATN activity of units that showed learning-related activity indicated that the earliest correlations occurred at $-10 \mathrm{~ms}$, with the highest correlations starting $-5 \mathrm{~ms}$ relative to CR onset (time lag 0) (Fig. 8C). Examination of simultaneously recorded spike-to-spike cross-correlations revealed in- 
A

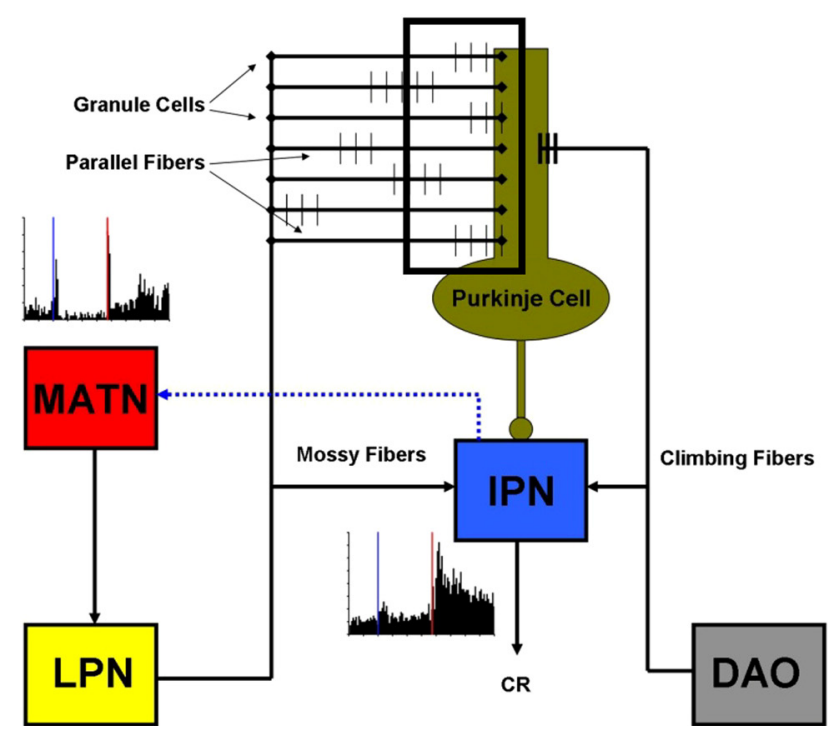

B

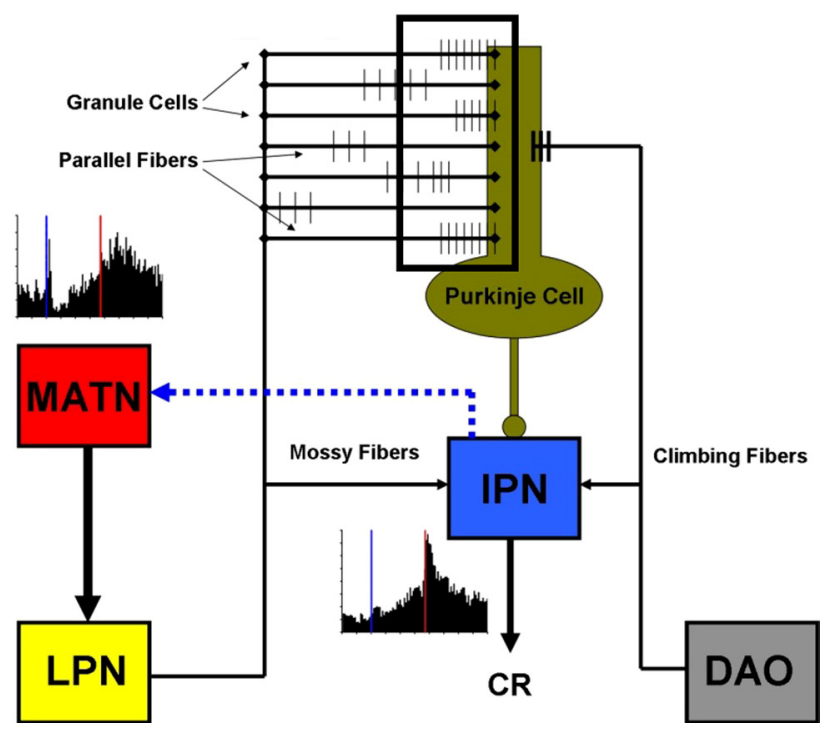

Figure 10. Hypothesized role of medial auditory thalamic plasticity in cerebellar learning. A, Paired stimulus inputs from the lateral pontine nuclei (LPN) and dorsal accessory inferior olive (DA0) on Purkinje cells in the cerebellar cortex during the early stages of eyeblink conditioning. Learning is initiated by CS activated parallel fibers that arrive at Purkinje cells nearly simultaneously with climbing fiber input (window). No learning-related plasticity is evident in the anterior IPN or MATN during the initial training trials. $\boldsymbol{B}$, Paired stimulus inputs from the LPN and DA0 on Purkinje cells after conditioned responding starts to emerge. Learning is potentiated by an increase (relative to initial training) in the frequency of parallel fiber activity at Purkinje cells that occurs nearly simultaneously with climbing fiber input (window). Clear learning-related plasticity is evident in IPN and MATN during this later stage of learning. An increase in learning-related excitatory cerebellar feedback to the MATN (blue dotted line) may in turn increase the MATN output to LPN and the corresponding mossy fiber projection into the cerebellum to further facilitate learning.

creased firing in IPN units preceded the increase observed in the MATN (Fig. 8D,E). Many more units in the IPN (72.6\%) showed significant cross-correlations at earlier lead times than MATN units (15.8\%) (Fig. 8 F). These results are consistent with the finding of significant correlations for the IPN population at $20 \mathrm{~ms}$ before CR onset, whereas the earliest correlations for each area of the MATN were immediately before CR onset $(-5 \mathrm{~ms})$. Overall, the results of the cross-correlation analyses indicate that learning-related unit activity occurs earlier within trials in the IPN than in the MATN.

\section{Fluoro-Gold retrograde tracing}

Fluoro-Gold was infused into the right MATN (Fig. 9B) to investigate axonal inputs that could play a role in the types of unit responses observed in the current experiment. Labeled neurons were observed in the contralateral cochlear nucleus, superior olive, nucleus of the lateral lemniscus, and inferior colliculus (Fig. $9 A$ ), as seen in previous reports (Henkel, 1983; Bajo et al., 1993; Malmierca et al., 2002; Coomes and Schofield, 2004). These auditory structures are the likely sources of CS input into the MATN. Labeled neurons were also found in the contralateral lateral vestibular nucleus and spinal trigeminal nucleus (Blum and Gilman, 1979; Bordi and LeDoux, 1994). Labeled neurons in the contralateral anterior IPN and dentate nucleus were also found (Fig. 9C). This cerebellar projection could be the source of the CRcorrelated neuronal responses observed in the MATN during CR retention.

\section{Discussion}

Learning-related changes in neuronal activity were observed in both the cerebellar interpositus nucleus and medial auditory thalamic nuclei during acquisition and extinction of delay eyeblink conditioning. Unit activity that correlated with the eyeblink CR developed in the IPN earlier than in the MATN across training sessions. The highest within-trial correlations between IPN activity and eyelid EMG occurred before CR onset, whereas the highest correlations between MATN activity and eyelid EMG occurred after CR onset. These findings suggest that CR-related increases in IPN activity could drive the CR-related increases in activity within the MATN, directly or indirectly. A neuroanatomical pathway that could provide feedback from the cerebellum to the MATN was demonstrated by retrograde tracer infusions into the MATN, which revealed a direct projection from the IPN and dentate nucleus to the MATN. The firing relationship between the cerebellum and thalamus and the cerebellar-MATN projection suggest that increases in CS evoked activity correlated with CR performance emerge in the IPN early in conditioning and are then reflected in the MATN through feedback. Alternatively, the CR-related changes in MATN activity could be established independently as a result of converging CS and US inputs (Edeline and Weinberger, 1992; Bordi and LeDoux, 1994). An argument against this alternative hypothesis is that cerebellar inactivation during acquisition blocks subsequent savings (Krupa et al., 1993; Freeman et al., 2005), which should be evident if the MATN undergo CR-related plasticity that is independent of the cerebellum.

Short-latency plasticity in the MATN developed early in training and was strongest in the MGm. This finding is similar to the results of a study that showed increased CS-elicited activity after fear conditioning in units that were responsive to the CS before training (Maren et al., 2001). There was also greater short-latency activity during trials with CRs relative to trials without CRs (Fig. 7). The short-latency CR-related activity was probably not directly influencing production of the CR because it subsided before the onset of the CR and was not seen during extinction even though CRs were still occurring. Greater activity in the MGm at the onset of conditioning trials may reflect increased attention. Increased attention could facilitate thalamic activity and thereby facilitate input to the cerebellum, increasing the probability of generating a CR.

Previous studies demonstrated that cerebellar feedback influences learning-related activity in afferent and efferent brain sys- 
tems. Lesions or inactivation of the IPN abolish both eyeblink CRs and learning-related activity in the PN, trigeminal nucleus, hippocampus, somatosensory cortex, ventrolateral thalamus, and red nucleus (Clark et al., 1984, 1997; Chapman et al., 1990; Clark and Lavond, 1996; Sears et al., 1996; Bao et al., 2000; Wikgren et al., 2006). The IPN has a direct excitatory projection to the $\mathrm{PN}$ and this projection is thought to facilitate plasticity in the PN mossy fiber input to the cerebellum over the course of learning (Bao et al., 2000). The cerebellar projection to the MATN may be playing a similar role. It is, therefore, possible that the plasticity observed in the MATN facilitates CS input to the PN, and subsequent CSspecific mossy fiber input to enhance synaptic plasticity within the cerebellum after acquisition has started. Facilitated CS input would then result in a steeper learning curve and a higher asymptotic level of conditioning.

Recent models of cerebellar learning posit that the long-term depression (LTD) developing at parallel fiber to Purkinje cell synapses in the cerebellar cortex releases the IPN from cortical inhibition, which in turn induces long-term potentiation (LTP) in the IPN (Mauk and Donegan, 1997; Medina and Mauk, 2000; Medina et al., 2000, 2002). According to these models, early in learning (i.e., the first session), the granule cells that are active during the end of the CS period participate in the induction of LTD in the cortex as they arrive at the Purkinje cell shortly before US input (Fig. 10A). Learning-related plasticity in the IPN begins to develop over the course of training as CS-specific LTD is established in the cortex (Medina et al., 2000). There are two important consequences of a CS-related increase in IPN activity: the occurrence of the eyeblink CR and increased excitatory cerebellar feedback to structures in the CS pathway including the MATN. Cerebellar feedback could increase the amount of activity in the MATN to the CS, which in turn would increase the output of the MATN to the PN for that particular CS (Fig. 10B). This would increase the mossy fiber input into the cerebellum associated with the CS used in training and strengthen synaptic plasticity, resulting in more rapid learning and a higher CR percentage (and amplitude) at asymptote. This thalamo-cerebellar positive-feedback loop could be a neural mechanism supporting learning theories that posit increased attention to the CS over the course of learning (Mackintosh, 1975).

Learning-related activity in the IPN and MATN was evident during acquisition but decreased during extinction training. The increase in inhibitory responses observed in the IPN for non-CR trials during extinction could provide clues regarding the neural mechanism underlying extinction. The increases in inhibitory responses in IPN during extinction may be attributable to increased inhibition from the cerebellar cortex caused by LTP at parallel fiber to Purkinje cell synapses (Mauk and Ohyama, 2004; Jirenhed et al., 2007). Cortical LTP may suppress LTP-related excitation in the IPN during CR trials and drive IPN activity below baseline on non-CR trials. The decrease in CS-elicited unit activity in the MATN during extinction may be a consequence of the decreased activity observed in the IPN and the resulting loss of positive feedback to the MATN. The LTP hypothesis is stimulus specific, but the decrease in baseline activity occurs in the absence of the CS. The decrease in baseline activity in IPN and MATN during extinction may be related to contextual control of extinction (Bouton and King, 1983). The hippocampus plays a critical role in context processing and may modulate baseline activity in the thalamus and cerebellum during extinction (Penick and Solomon, 1991; Freeman et al., 1996; Maren and Fanselow, 1997; Frohardt et al., 2000; Ji and Maren, 2008). Hippocampal activity can modulate cerebellar activity by synchronizing theta in both structures during acquisition of eyeblink conditioning (Hoffmann and Berry, 2009). Contextual processing in the hippocampus during extinction training could similarly influence the cerebellum and result in inhibition of baseline activity.

Overall, this study demonstrates that neurons in both the interpositus nucleus and medial auditory thalamus show increases in firing correlated with the eyeblink CR, but interpositus nucleus neurons show learning-related activity that substantially precedes learning-related activity in the medial auditory thalamus across training sessions and within training trials. The learningrelated increase in activity within the interpositus nucleus may produce the learning-related activity in the medial auditory thalamus through a direct positive-feedback projection. The medial auditory thalamus is a necessary relay of auditory CS information to the pontine nuclei (Halverson and Freeman, 2006, 2010; Campolattaro et al., 2007; Halverson et al., 2008) and may play a role in the learning process by increasing CS-related input to the pontine nuclei, thereby boosting mossy fiber input to the cerebellum and facilitating the induction of synaptic plasticity.

\section{References}

Bajo VM, Merchán MA, López DE, Rouiller EM (1993) Neuronal morphology and efferent projections of the dorsal nucleus of the lateral lemniscus in the rat. J Comp Neurol 334:241-262.

Bao S, Chen L, Thompson RF (2000) Learning- and cerebellum-dependent neuronal activity in the lateral pontine nucleus. Behav Neurosci 114:254-261.

Berthier NE, Moore JW (1990) Activity of deep cerebellar nucleus cells during classical conditioning of nictitating membrane extension in rabbits. Exp Brain Res 83:44-54.

Blum PS, Gilman S (1979) Vestibular, somatosensory, and auditory input to the thalamus of the cat. Exp Neurol 65:343-354.

Bordi F, LeDoux JE (1994) Response properties of single units in areas of rat auditory thalamus that project to the amygdala. Exp Brain Res 98:275-286.

Bouton ME, King DA (1983) Contextual control of the extinction of conditioned fear: tests for the associative value of the context. J Exp Psychol Anim Behav Process 9:248-265.

Calford MB, Aitkin LM (1983) Ascending projections to the medial geniculate body of the cat: evidence for multiple, parallel auditory pathways through thalamus. J Neurosci 3:2365-2380.

Campolattaro MM, Halverson HE, Freeman JH 2007 Medial auditory thalamic stimulation as a conditioned stimulus for eyeblink conditioning in rats. Learn Mem 14:152-159.

Cartford MC, Gohl EB, Singson M, Lavond DG (1997) The effects of reversible inactivation of the red nucleus on learning-related and auditoryevoked unit activity in the pontine nuclei of classically conditioned rabbits. Learn Mem 3:519-531.

Chapman PF, Steinmetz JE, Sears LL, Thompson RF (1990) Effects of lidocaine injection in the interpositus nucleus and red nucleus on conditioned behavioral and neuronal responses. Brain Res 537:149-156.

Choi JS, Moore JW (2003) Cerebellar neuronal activity expresses the complex topography of conditioned eyeblink response. Behav Neurosci 117:1211-1219.

Christian KM, Thompson RF (2003) Neural substrates of eyeblink conditioning: acquisition and retention. Learn Mem 11:427-455.

Clark GA, McCormick DA, Lavond DG, Thompson RF (1984) Effects of lesions of cerebellar nuclei on conditioned behavioral and hippocampal neuronal responses. Brain Res 291:125-136.

Clark RE, Lavond DG (1996) Neural unit activity in the trigeminal complex with interpositus or red nucleus inactivation during classical eyeblink conditioning. Behav Neurosci 110:13-21.

Clark RE, Zhang AA, Lavond DG (1997) The importance of cerebellar cortex and facial nucleus in acquisition and retention of eyeblink/NM conditioning: evidence for critical unilateral regulation of the conditioned response. Neurobiol Learn Mem 67:96-111.

Coomes DL, Schofield BR (2004) Separate projections from the inferior colliculus to the cochlear nucleus and thalamus in guinea pigs. Hear Res 191:67-78.

Disterhoft JF, Olds J (1972) Differential development of conditioned unit changes in thalamus and cortex of rat. J Neurophysiol 35:665-679. 
Edeline JM, Weinberger NM (1992) Associative retuning in the thalamic source of input to the amygdala and auditory cortex: receptive field plasticity in the medial division of the medial geniculate body. Behav Neurosci 106:81-105.

Freeman JH Jr, Nicholson DA (1999) Neuronal activity in the cerebellar interpositus and lateral pontine nuclei during inhibitory classical conditioning of the eyeblink response. Brain Res 833:225-233.

Freeman JH Jr, Nicholson DA (2000) Developmental changes in eye-blink conditioning and neural activity in the cerebellar interpositus nucleus. J Neurosci 20:813-819.

Freeman JH Jr, Cuppernell C, Flannery K, Gabriel M (1996) Contextspecific multi-site cingulate cortical, limbic thalamic, and hippocampal neuronal activity during concurrent discriminative approach and avoidance training in rabbits. J Neurosci 16:1538-1549.

Freeman JH Jr, Halverson HE, Poremba A (2005) Differential effects of cerebellar inactivation on eyeblink conditioned excitation and inhibition. J Neurosci 25:889-895.

Frohardt RJ, Guarraci FA, Bouton ME (2000) The effects of neurotoxic hippocampal lesions on two effects of context after fear extinction. Behav Neurosci 114:227-240.

Gabriel M, Saltwick SE, Miller JD (1975) Conditioning and reversal of short-latency multiple-unit responses in the rabbit medial geniculate nucleus. Science 189:1108-1109.

Gould TJ, Steinmetz JE (1996) Changes in rabbit cerebellar cortical and interpositus nucleus activity during acquisition, extinction and backward classical conditioning. Neurobiol Learn Mem 65:17-34.

Green JT, Arenos JD (2007) Hippocampal and cerebellar single-unit activity during delay and trace eyeblink conditioning in the rat. Neurobiol Learn Mem 87:269-284.

Green JT, Johnson TB, Goodlett CR, Steinmetz JE (2002) Eyeblink classical conditioning and interpositus nucleus activity are disrupted in adult rats exposed to ethanol neonates. Learn Mem 9:304-320.

Halverson HE, Freeman JH (2006) Medial auditory thalamic nuclei are necessary for eyeblink conditioning. Behav Neurosci 120:880-887.

Halverson HE, Freeman JH (2010) Medial auditory thalamic input to the lateral pontine nuclei is necessary for auditory eyeblink conditioning. Neurobiol Learn Mem 93:92-98.

Halverson HE, Poremba A, Freeman JH (2008) Medial auditory thalamus inactivation prevents acquisition and retention of eyeblink conditioning. Learn Mem 15:532-538.

Henkel CK (1983) Evidence of sub-collicular auditory projections to the medial geniculate nucleus in the cat: an autoradiographic and horseradish peroxidase study. Brain Res 259:21-30.

Hoffmann LC, Berry SD (2009) Cerebellar theta oscillations are synchronized during hippocampal theta-contingent trace conditioning. Proc Natl Acad Sci U S A 106:21371-21376.

Ji J, Maren S (2008) Differential roles for hippocampal areas CA1 and CA3 in the contextual encoding and retrieval of extinguished fear. Learn Mem 15:244-251.

Jirenhed DA, Bengtsson F, Hesslow G (2007) Acquisition, extinction, and reacquisition of a cerebellar cortical memory trace. J Neurosci 27:2493-2502.

Krupa DJ, Thompson JK, Thompson RF (1993) Localization of a memory trace in the mammalian brain. Science 260:989-991.

Kubota Y, Wolske M, Poremba A, Kang E, Gabriel M (1996) Stimulusrelated and movement-related single-unit activity in rabbit cingulated cortex and limbic thalamus during performance of discriminative avoidance behavior. Brain Res 721:22-38.

LeDoux JE, Ruggiero DA, Reis DJ (1985) Projections to the subcortical forebrain from anatomically defined regions of the medial geniculate body in the rat. J Comp Neurol 242:182-213.

LeDoux JE, Ruggiero DA, Forest R, Stornetta R, Reis DJ (1987) Topographic organization of convergent projections to the thalamus from the inferior colliculus and spinal cord in the rat. J Comp Neurol 264:123-146.

Mackintosh NJ (1975) A theory of attention: variations in the associability of stimuli with reinforcement. Psychol Rev 82:276-298.

Malmierca MS, Merchán MA, Henkel CK, Oliver DL (2002) Direct projections from cochlear nuclear complex to auditory thalamus in the rat. J Neurosci 22:10891-10897.

Maren S, Fanselow MS (1997) Electrolytic lesions of the fimbria/fornix, dorsal hippocampus, or entorhinal cortex produce anterograde deficits in contextual fear conditioning in rats. Neurobiol Learn Mem 67:142-149.
Maren S, Yap SA, Goosens KA (2001) The amygdala is essential for the development of neuronal plasticity in the medial geniculate nucleus during auditory fear conditioning in rats. J Neurosci 21:RC135(1-6).

Mauk MD, Donegan NH (1997) A model of pavlovian eyelid conditioning based on the synaptic organization of the cerebellum. Learn Mem 4:130-158.

Mauk MD, Ohyama T (2004) Extinction as new learning versus unlearning: considerations from a computer simulation of the cerebellum. Learn Mem 11:566-571.

Mauk MD, Steinmetz JE, Thompson RF (1986) Classical conditioning using stimulation of the inferior olive as the unconditioned stimulus. Proc Natl Acad Sci U S A 83:5349-5353.

McCormick DA, Thompson RF (1984a) Cerebellum: essential involvement in the classically conditioned eyelid response. Science 223:296-299.

McCormick DA, Thompson RF (1984b) Neuronal responses of the rabbit cerebellum during acquisition and performance of a classically conditioned nictitating membrane-eyelid response. J Neurosci 4:2811-2822.

McCormick DA, Clark GA, Lavond DG, Thompson RF (1982) Initial localization of the memory trace for a basic form of learning. Proc Natl Acad Sci U S A 79:2731-2735.

Medina JF, Mauk MD (2000) Computer simulation of cerebellar information processing. Nat Neurosci 3:1205-1211.

Medina JF, Garcia KS, Nores WL, Taylor NM, Mauk MD (2000) Timing mechanisms in the cerebellum: testing predictions of a large-scale computer simulation. J Neurosci 20:5516-5525.

Medina JF, Nores WL, Mauk MD (2002) Inhibition of climbing fibers is a signal for the extinction of conditioned eyelid responses. Nature 416:330-333.

Nicholson DA, Freeman JH Jr (2002) Neuronal correlates of conditioned inhibition of the eyeblink response in the anterior interpositus nucleus. Behav Neurosci 116:22-36.

Nicholson DA, Freeman JH Jr (2003a) Addition of inhibition in the olivocerebellar system and the ontogeny of a motor memory. Nat Neurosci 6:532-537.

Nicholson DA, Freeman JH Jr (2003b) Developmental changes in evoked Purkinje cell complex spike responses. J Neurophysiol 90:2349-2357.

Nolan BC, Freeman JH (2006) Purkinje cell loss by OX7-saporin impairs acquisition and extinction of eyeblink conditioning. Learn Mem 13:359-365.

O'Connor KN, Allison TL, Rosenfield ME, Moore JW (1997) Neural activity in the medial geniculate nucleus during auditory trace conditioning. Exp Brain Res 113:534-556.

Ohyama T, Nores WL, Medina JF, Riusech FA, Mauk MD (2006) Learninginduced plasticity in deep cerebellar nucleus. J Neurosci 26:12656-12663.

Paxinos G, Watson C (1998) The rat brain in stereotaxic coordinates. New York: Academic.

Penick S, Solomon PR (1991) Hippocampus, context, and conditioning. Behav Neurosci 105:611-617.

Poremba A, Gabriel M (2001) Amygdalar efferents initiate auditory thalamic discriminative training-induced neuronal activity. J Neurosci 21:270-278.

Ryugo DK, Weinberger NM (1978) Differential plasticity of morphologically distinct neuron populations in the medial geniculate body of the cat during classical conditioning. Behav Biol 22:275-301.

Sears LL, Steinmetz JE (1990) Acquisition of classically conditioned-related activity in the hippocampus is affected by lesions of the cerebellar interpositus nucleus. Behav Neurosci 104:681-692.

Sears LL, Logue SF, Steinmetz JE (1996) Involvement of the ventrolateral thalamic nucleus in rabbit classical eyeblink conditioning. Behav Brain Res 74:105-117.

Steinmetz JE, Logan CG, Rosen DJ, Thompson JK, Lavond DG, Thompson RF (1987) Initial localization of the acoustic conditioned stimulus projection system to the cerebellum essential for classical eyelid conditioning. Proc Natl Acad Sci U S A 84:3531-3535.

Steinmetz JE, Lavond DG, Thompson RF (1989) Classical conditioning in rabbits using pontine nucleus stimulation as a conditioned stimulus and inferior olive stimulation as a unconditioned stimulus. Synapse 3:225-233.

Supple WF Jr, Kapp BS (1989) Response characteristics of neurons in the medial component of the medial geniculate nucleus during pavlovian differential fear conditioning in rabbits. Behav Neurosci 103:1276-1286.

Wikgren J, Lavond DG, Ruusuvirta T, Korhonen T (2006) Cooling of the cerebellar interpositus nucleus abolishes somatosensory cortical learningrelated activity in eyeblink conditioned rabbits. Behav Brain Res 170:94-98. 